\title{
Comparison of magnetic- and chemical-boundary roughness in magnetic films and multilayers
}

\author{
J. J. Kelly IV, B. M. Barnes, F. Flack, D. P. Lagally, D. E. Savage, M. Friesen, \\ and M. G. Lagallya) \\ University of Wisconsin-Madison, Madison, Wisconsin 53706
}

(Received 19 November 2001; accepted for publication 21 March 2002)

\begin{abstract}
Diffuse x-ray resonant magnetic scattering, atomic-force microscopy, and magnetic hysteresis measurements are used to explore the relationship between the roughness and magnetic properties of interfaces between magnetic and nonmagnetic thin films. Bare Co films and Co films capped with magnetic and nonmagnetic thin films are investigated to elucidate why and under what circumstances the magnetic boundary differs from the chemical boundary. Competing models to explain why the magnetic boundary appears smoother than the chemical boundary are explored.

(c) 2002 American Institute of Physics. [DOI: 10.1063/1.1478142]
\end{abstract}

\section{INTRODUCTION}

Technology to read and write bits for magnetic data storage has been evolving rapidly. Read heads in modern hard drives use the magnetoresistance or giant magnetoresistance (GMR) effect rather than the older inductive methods. Nonvolatile memory based on GMR is also of current interest. A key component of such magnetoelectronic devices is a single, thin magnetic film or stack of thin magnetic films with nonmagnetic spacer layers, with layer thicknesses usually less than $10 \mathrm{~nm}$. For these films, interfaces become critically important. In magnetic films in contact with nonmagnetic ones one can describe separately a chemical boundary and a magnetic boundary between the two materials. The chemical boundary simply lies between the atoms of the two materials. The magnetic boundary, defined for the magnetic material, separates those atoms that follow the applied magnetic field (the "bulk" atoms) from those that do not. The magnetic boundary need not, in general, follow the chemical boundary. If it does not coincide with the chemical boundary, it must lie more in the magnetic material, i.e., an interface region, sometimes inappropriately called a dead layer, in which the magnetic moments do not perfectly follow the applied field, is implied. The width of this magnetic interface region presumably depends on the magnitude of the applied field, the temperature, and possibly also the thickness of the magnetic film.

The topography defined by the chemical boundary (e.g., roughness, wavelength of undulations, etc.) can apparently play a role in defining thin-film magnetic properties. In-plane magnetization reversal processes, magnetic anisotropy, ${ }^{1,2}$ and spin dependent scattering depend on the chemical-boundary morphology. Chemical-boundary morphology may also affect exchange bias $^{3}$ in some systems, for example antiferromagnetic-ferromagnetic bilayers, with evidence that the underlying physical mechanism is related to uncompensated spins present at the ferromagnetic-antiferromagnetic interface caused by structural disorder at the interface. ${ }^{4} \mathrm{~A}$ de-

\footnotetext{
a)Electronic mail: lagally@engr.wisc.edu
}

tailed understanding of the influence of surface and interface morphology on these thin-film magnetic properties is lacking. Several studies, both theoretical and experimental, have examined the effect of interface morphology on spindependent scattering by measuring the GMR of films with varying degrees of interfacial roughness. ${ }^{5-11}$ Two studies ${ }^{7,11}$ find that the GMR increases with increasing roughness, while one ${ }^{6}$ finds that the GMR decreases with increasing roughness. Others ${ }^{8,10}$ find no influence of roughness, but rather that bulk scattering ${ }^{8}$ or crystallographic texture ${ }^{10}$ dominates.

It is difficult to characterize the morphology of buried interfaces. A scanned-probe microscopy measurement of the nanomorphology of surfaces may not reflect that of a buried interface, because the internal boundaries may be modified by interdiffusion and chemical reaction. Although crosssectional transmission electron microscopy can image buried interfaces and is sensitive to the chemical interface, it is destructive, has a limited bandwidth of roughness length scales that can be probed, and averages over depth along the direction of viewing. X-ray scattering, and in particular diffuse $\mathrm{x}$-ray scattering (DXRS), is more powerful; it is nondestructive and can be used to quantify buried-interface morphology under appropriate conditions. Morphology can be measured with $\mathrm{x}$ rays in two ways. One can measure the specularly reflected intensity as both the sample and the detector are moved together. This approach provides information about layer thicknesses, composition, and total roughness. Alternatively, either the sample or the detector may be fixed while the other moves. With this approach one can measure both the specularly scattered intensity and the diffusely scattered intensity, providing information about the lateral distribution of the roughness, as well as the total roughness. In all the studies mentioned earlier, ${ }^{5-11}$ the interfacial morphology was measured by specular x-ray scattering. DXRS is also sensitive to the chemical interface between two media, i.e., the atoms of one material can be distinguished from those of the other, if the wavelength of the $\mathrm{x}$ rays can be varied.

The magnetic boundary may differ from the chemical boundary. There may be magnetic moments in the chemical- 
interface region (in which both magnetic and nonmagnetic atoms are present) that do not contribute to the bulk magnetism. Thus, a probe that is sensitive to magnetic moments, such as neutrons or polarized $\mathrm{x}$ rays, is needed to explore possible differences in the morphology of magnetic and chemical boundaries. Neutron scattering studies provided the first evidence of differing magnitudes of the chemical and the magnetic roughness. The decrease of the specular reflectivity, caused by interfacial roughness, was less for magnetic scattering than for chemical scattering, suggesting a magnetic boundary that is smoother than the chemical boundary. ${ }^{12,13}$ To gain information on the lateral distribution of the interfacial roughness, one would need a measurement of the diffusely scattered neutrons, something that is currently impossible because of the low signal.

Direct evidence that the magnetic and chemical boundary roughnesses can differ has been obtained using diffuse soft-x-ray resonant magnetic scattering (DXRMS) ${ }^{14}$ measurements of a Co thin film capped with Al. DXRMS uses circularly polarized photons, tuned to the absorption edge of the magnetic material, to obtain an enhanced magnetic component of the x-ray scattering. In these measurements, in which the in-plane magnetic field is switched as the magnetic diffuse scattering in measured, the roughness of the magnetic boundary was lower than that of the chemical boundary, and the height-height correlation length of the magnetic roughness was greater than that of the chemical roughness. ${ }^{14}$ Three possible models were postulated to explain the observations. One, the moments could be pinned in the magnetic-interface region, thus preventing them from rotating with the applied field. Such pinning could result from an anisotropy at the interface that makes out-of-plane alignment energetically favorable. ${ }^{15}$ Two, the moments could be chemically quenched in the interface region, thus reducing the magnitude of the magnetization, $M$, for the interface moments. Three, a reduced exchange coupling of moments in the interface region may exist. Moments at a disordered interface have lower coordination, and lower coordination could cause interface moments with fewer nearest neighbors to have a reduced exchange coupling to moments in the bulk. Models 1 and 3 both predict that moments do not follow the applied magnetic field, but in one case the moments are frozen in place, and in the other they are "flopping about." A recent theoretical paper predicts "loose moments" (model 3) using a simple Ising model. ${ }^{16}$

Differences between magnetic- and chemical-boundary roughness have recently been observed for two additional magnetic-thin-film systems. In CoFe alloy films capped with $\mathrm{Cu}$ the magnetic boundary is smoother than the chemical boundary, and the height-height correlation lengths of the magnetic roughness were longer than that of the chemical roughness, in agreement with Ref. $14 .{ }^{17}$ The roughness and correlation length relationships are also the same for a more complicated system, Fe/Gd multilayers, where the Gdspecific magnetic boundary in contact with the $\mathrm{Fe}$ is measured. ${ }^{18} \mathrm{Gd}$ has no bulk ferromagnetism, and only gains ferromagnetic order when in contact with a ferromagnet.

A theory developed subsequent to early measurements ${ }^{14,17}$ reveals that what had been identified in those measurements as purely magnetic scattering was actually a crosscorrelation of magnetic and chemical scattering. ${ }^{19}$ A subsequent study of $\mathrm{Co} / \mathrm{Cu}$ multilayers using scattering of linearly polarized $\mathrm{x}$ rays to measure magnetic roughness at the $\mathrm{Co} / \mathrm{Cu}$ interface, a method that is free from chargemagnetic correlations, shows ${ }^{20}$ much longer height-height correlation lengths for the magnetic-boundary roughness than for the chemical boundary (agreeing qualitatively in this aspect with earlier measurements) ${ }^{14,17,18}$ but no significant difference between the magnitudes of the rms roughnesses of the magnetic and chemical boundaries (in contrast to earlier measurements). ${ }^{14,17,18}$ This partial agreement with earlier results may be a function of how the films were grown (see later) and not due to the method of analysis. No efforts were made to compare chemical roughnesses using the two approaches (linearly and circularly polarized $\mathrm{x}$ rays) but it is clear that even more precise analysis does not eliminate differences in morphology observed for magnetic and chemical boundaries.

The studies described earlier have examined magnetic materials in contact with nonmagnetic ones (except the $\mathrm{Fe} / \mathrm{Gd}$ multilayers, see discussion earlier). Such studies cannot eliminate any of the three models described earlier. In this article, we attempt to address the differences between the models by measuring the diffuse XRMS from a clean, bare Co film and an Fe-capped Co film, and comparing these results with our earlier studies on Al-capped Co films. Examining a bare Co film frees us from the complications of an overlayer, eliminating the possibility of quenching by a nonmagnetic cap layer. By covering a Co film with Fe, we change the problem from a magnetic/nonmagnetic interface to a magnetic/magnetic interface. If there is some loss of magnetic order at a bare Co surface due to reduced exchange coupling, the strongly ferromagnetic Fe will compensate for it. The Fe cap layer is also chemically similar to Co, thus any possible anisotropy at the bare Co surface that pins moments in an out-of-plane orientation may be affected by the Fe cap layer.

We observe differences in the nature of the magnetic boundary for these situations. The bare Co surface behaves similarly to the Al-capped Co film. Fe capping brings the roughness of the Co magnetic boundary very close to that of the chemical boundary between $\mathrm{Fe}$ and $\mathrm{Co}$.

\section{EXPERIMENT}

The experiments were performed at the Synchrotron Radiation Center of the University of Wisconsin-Madison using a beamline with a $\mathrm{C} / \mathrm{W}$ multilayer mirror monochromator that delivers $\sim 6 \times 10^{8}$ elliptically polarized photons/sec to the sample at $\sim 775 \mathrm{eV}$, the $L_{3}$ edge of $\mathrm{Co}$, at $\sim 710 \mathrm{eV}$, the $L_{3}$ edge for $\mathrm{Fe}$, and potentially other edges of interest in this vicinity. The energy resolution, $\Delta \lambda / \lambda$, is $4 \%$, or approximately $30 \mathrm{eV}$ at $780 \mathrm{eV}$. Good energy resolution is unimportant in these measurements, as long as one can distinguish different absorption edges. By tuning the monochromator to other energies, other absorption edges can be reached. Films were deposited using dc magnetron sputtering at $2.0 \mathrm{mTorr}$ Ar pressure in an UHV chamber with $\sim 5 \times 10^{-10}$ Torr base 


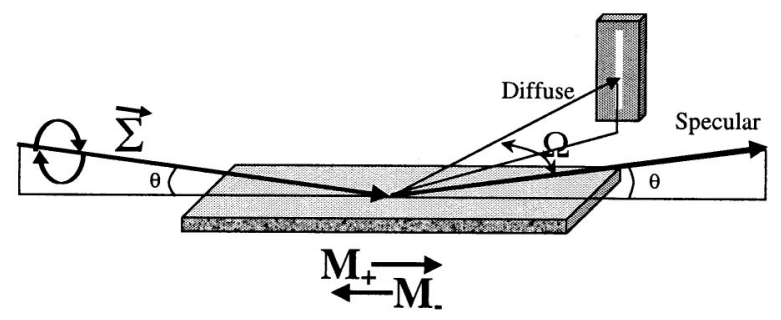

FIG. 1. The geometry of the DXRMS experiment. The sample stays fixed during a scan while the detector moves out of the scattering plane.

pressure directly connected to the analysis chamber. Sputter deposited films are in general smoother than evaporated films of comparable thickness, because of the action of the plasma in reducing asperities during growth. Films with chemical roughness of as little as $0.3 \mathrm{~nm}$ rms are routinely used in commercial read-write heads. Use of sputter deposited films matches industrial practice and sets the most stringent conditions for magnetic-interface roughness studies. The samples are transferred without breaking vacuum to the diffractometer chamber. Three types of samples are compared: bare Co $(7.0 \mathrm{~nm} \mathrm{Co} / \mathrm{Si}(100)$, Fe capped Co $(2.0 \mathrm{~nm}$ $\mathrm{Fe} / 7.0 \mathrm{~nm} \mathrm{Co} / \mathrm{Si}(100)$, and $\mathrm{Al}$ capped Co $(2.5 \mathrm{~nm} \mathrm{Al} / 7.0 \mathrm{~nm}$ $\mathrm{Co} / \mathrm{Si}(100)$. The $2.0 \mathrm{~nm} \mathrm{Fe} / 7.0 \mathrm{~nm} \mathrm{Co} / \mathrm{Si}(100)$ sample was sputter deposited using the same parameters for the Co layer as the $7.0 \mathrm{~nm} \mathrm{Co} / \mathrm{Si}(100)$ sample.

We measure the scattered $\mathrm{x}$ rays as a function of the angle $\Omega$ orthogonal to the scattering plane ${ }^{21,22}$ (Fig. 1) to obtain not just the specularly reflected intensity, but the diffuse intensity as far out in reciprocal space as we can see intensity, roughly $\pm 40^{\circ}$ from the specular peak, which allows us to see roughness correlations down to lateral length scales of $20 \AA$. Such a scan in $\Omega$ is termed an azimuthal transverse scan. The azimuthal transverse scans are done at a fixed $3^{\circ}$ angle of incidence, maximizing reflectance by staying in the regime of total external reflection. The scattered intensity is measured using a channel electron multiplier with a Si photodiode as the photocathode, masked by a slit $0.7 \mathrm{~mm}$ wide by $10 \mathrm{~mm}$ high. The intensity in the scattering plane $\left(S_{z}\right)$ is therefore integrated. We utilize this technique for two main reasons. One, the range of roughness wavelengths contributing to the measured rms roughness is the broadest possible, extending from the wavelength of the $\mathrm{x}$ rays $(\sim 1.5 \mathrm{~nm})$ to $\sim 1 \mu \mathrm{m}$, limited only by detector design at the long wavelength. Thus, the bandwidth for roughness measurements is very wide. Two, this measurement geometry simplifies the modeling. ${ }^{23}$

We magnetize the sample with an electromagnet that delivers a maximum field of $300 \mathrm{G}$ either parallel or antiparallel to the photon helicity. We generally use fields of $\sim 50-$ $100 \mathrm{G}$, about 5-10 times the coercive field of our samples. We measure the scattered intensity point by point for magnetization parallel and antiparallel to the photon helicity, called $I_{+}$and $I_{-}$, respectively. We then calculate the difference intensity, $\Delta I(M, \Omega)=I_{+}-I_{-}$, and the sum intensity, $I_{\text {ave }}(\Omega)=(1 / 2)\left(I_{+}+I_{-}\right)$. A scan from a demagnetized sample would have an intensity of $(1 / 2)\left(I_{+}+I_{-}\right)$, i.e., between a full scan taken with magnetization parallel to the photon helicity, and a scan taken with the magnetization antiparallel. $\Delta I(M, \Omega)$ and $I_{\text {ave }}(\Omega)$ are normalized to one at the specular peak intensity $(\Omega=0)$. We use the normalization to compare the relative shapes of the specular and diffuse intensities of $\Delta I(M, \Omega)$ and $I_{\text {ave }}(\Omega)$, which reflect the interface roughness parameters as detailed later.

In addition to the DXRMS measurements, we obtain element specific hysteresis curves using the XRMS specular reflection to determine the coercive force and magnetic quality of the films. We can also use the diffuse intensity to perform element specific hysteresis measurements, giving us greater sensitivity to the magnetic quality of the interface.

Although we have not done so for these samples, it is also possible to measure the asymmetry ratio, the intensity when the sample is magnetized in one direction minus the intensity when the sample is magnetized in the opposite direction, divided by their average. Its value for a given element depends on the relative number of moments that follow the applied field. In the limit that a film is nonmagnetic or for some reason entirely pinned, the asymmetry ratio is zero, i.e., there is no change in intensity when the direction of the field is reversed. The asymmetry ratio can depend quite strongly on the angle of incidence. ${ }^{24}$

We measure surface roughness of exterior surfaces (i.e., for the bare Co film and the Fe surface of the Fe-capped Co film) with atomic-force microscopy (AFM) in the intermittent-contact mode, using carbon nanotube (CNT) probes for enhanced resolution. ${ }^{25}$ Measurements made with CNTs show a larger rms roughness than those made with regular Si etched tips; nevertheless we expect AFM to produce slightly lower rms roughness values than do $\mathrm{x}$ rays because of the wider range of frequencies that the $\mathrm{x}$ rays can see. Additionally, because these measurements are ex situ, the surfaces will be slightly oxidized. We expect that the roughness changes somewhat upon oxidization, with the oxide preferentially transforming shorter to longer-wavelength roughness.

We determine interface roughness from the x-ray scattering measurements by modeling the diffuse and specular components of the scattering, obtaining information on both the chemical and magnetic disorder. A perfectly smooth interface or surface would result in only a specular peak. Roughness scatters $\mathrm{x}$ rays away from the specular direction into a diffuse halo. In the specular direction both the specular and diffuse components are superimposed, although the specular component is typically very much larger than the diffuse component.

The surface morphology of thin films can generally be described by a Gaussian self-affine fractal with a longwavelength cutoff. ${ }^{26}$ Such a model gives a surface heightheight correlation function of

$$
C(R)=\sigma^{2} \exp \left[-\left(\frac{R}{\xi}\right)^{2 h}\right],
$$

where $\sigma$ is the root mean square amplitude of the surface or interface roughness, $\xi$, the correlation length, is a measure of the lateral length scale of the roughness and $h$, the Hurst parameter, describes the texture of the roughness, i.e., roughness with a large $h$ would look like smooth sand dunes, while 
roughness with a small $h$ would be rocky, broken ground. ${ }^{26}$ Even though the overall topology (rolling hills) may look similar, on short scales surfaces with large and small $h$ look very different. Fitting the diffusely scattered intensity allows one to extract these parameters.

The scattering amplitude of circularly polarized $x$ rays at the $L_{2}$ or $L_{3}$ edge of a transition element contains the usual charge scattering term plus a resonant magnetic-scattering term that depends on the magnetization $M^{27}$ and changes sign when the magnetization is reversed, i.e.,

$$
I_{ \pm}=I_{\text {charge }}+I_{\text {resonant }}\left(M_{ \pm}\right) .
$$

Here $I_{\text {resonant }}\left(M_{ \pm}\right)$refers to the component of the intensity that changes with the reversal of the magnetization. $I_{\text {charge }}$ does not change with the reversal of the magnetization. The difference $I_{+}-I_{-}=\Delta I(M)$, contains the element specific resonant contribution to the scattered intensity. $\Delta I(M)$ is element specific because it is large only for scattering from magnetic atoms with an absorption edge corresponding to the selected photon energy. $\Delta I(M)$ will have a specular and diffuse component. The morphology of the magnetic boundary (either interface or surface) is reflected in the shape and relative intensity of the diffuse component $\Delta I(M, \Omega)_{\text {diffuse }}$.

To compare relatively the morphologies of different films in a quantitative manner, we use the distorted-wave Born approximation (DWBA). The amplitude scattered from the rough surface can be written as ${ }^{23}$

$$
\Psi_{s}=-\frac{e^{i k_{0} r}}{4 \pi r} k_{c}^{2} \mathfrak{J}_{i} \mathfrak{J}_{f} \int d^{2} r r_{\|}^{\prime} e^{-i q_{\|} r_{\|}^{\prime}} \int_{0}^{h\left(r_{\|}^{\prime}\right)} d z^{\prime} e^{-i q_{\perp} z}
$$

Because the incident angle does not change in our experiment, we perform our fitting with $q_{\perp}$ assumed to be a constant. The factors outside the integrals depend only on $\theta$. By normalizing our data, we eliminate the need to determine these factors. Fitting the normalized diffuse charge intensities, $I_{\text {charge }}$ in Eq. (2), produces a unique, physically defined triplet of the parameters $\sigma, \xi, h$ described earlier. The interpretation of the difference scattering $\Delta I(M)$ in terms of quantitative physical values requires application of the more recent theory, ${ }^{18,19}$ which describes how the magnetic layer alters the scattering of polarized photons. It shows that the dominant term contributing to the difference signal depends on the crosscorrelation of the morphologies of the magnetic and chemical interfaces. The theory ${ }^{18,19}$ has not yet been extended to the DWBA, and therefore interpretation of absolute scattered intensities is not possible. Interpreting the shape of the scattered intensity distribution allows us, however, to obtain the statistical parameters that describe the chemical boundary, independent of the limitation on determining absolute intensities. A similar approach can be applied to the difference intensity distribution to describe the magnetic boundary, but extracting the precise morphology of the magnetic interface depends on knowing the crosscorrelation between the chemical and magnetic boundaries.

\section{RESULTS}

AFM generally shows film surfaces having a shortwavelength roughness with a lateral correlation length on the

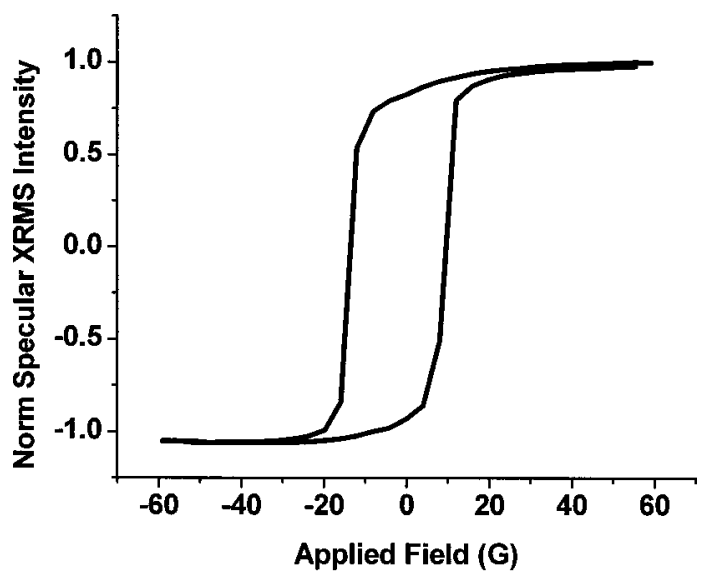

FIG. 2. Measurement of the element-specific hysteresis of sputter deposited bare, clean $\mathrm{Co}(7.0 \mathrm{~nm} \mathrm{Co} / \mathrm{Si}(100)$ films. The coercive field is $12 \mathrm{G}$ and the loop is nearly square, suggesting a single-domain film.

order of 10-100 $\mathrm{nm}$, and a rms roughness averaged over a 1 $\mu \mathrm{m}$ square of the order of a few angstroms. On large length scales the films are very smooth. Detailed values are given below when we discuss the individual samples.

Examples of element-specific hysteresis curves are shown in Figs. 2 and 3. The bare-Co/Si(100) film has a nearly square hysteresis loop, $S \sim 1$ (Fig. 2), indicating a single-domain sample, and a coercive field of about $12 \mathrm{G}$. We define squareness as $S=M_{B=0} / M_{\text {saturation. The } \mathrm{Fe} / \mathrm{Co} /}$ $\mathrm{Si}(100)$ film shows lower squareness $S \sim 0.5$ (Fig. 3), and may have multiple domains. It has a coercive field of about $10 \mathrm{G}$.

Figure 4 compares $\Delta I(M, \Omega)$ and $I_{\text {ave }}(\Omega)$ azimuthal scans for the clean Co surface $[7.0 \mathrm{~nm} \mathrm{Co/Si}(100)]$ using $\mathrm{x}$ rays tuned to the Co $L_{3}$ edge $(775 \mathrm{eV})$. This sample was grown in UHV, and the scattering data were taken within 30 $\mathrm{h}$, in the same UHV system. We expect less than $1 \mathrm{ML}$ of impurities adsorbed on the surface in this time. DXRMS shows that there is a significant difference in roughness of

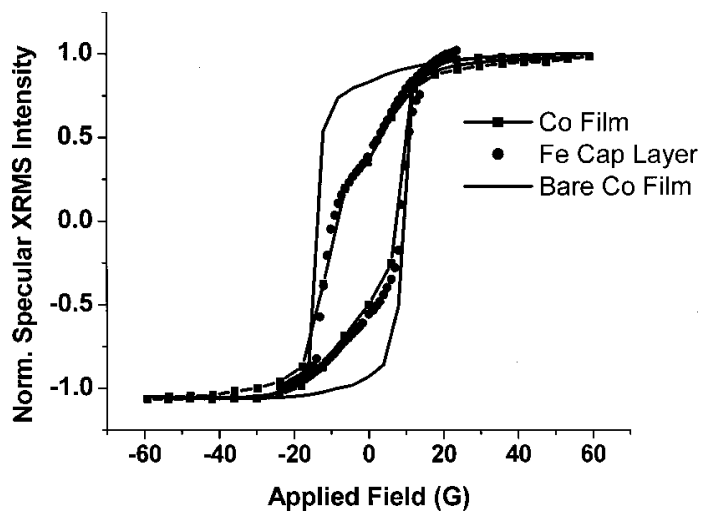

FIG. 3. Measurement of the element-specific hysteresis of Fe-capped Co $[2.0 \mathrm{~nm} \mathrm{Fe} / 7.0 \mathrm{~nm} \mathrm{Co} / \mathrm{Si}(100)]$ films. The squares (with a line to guide the eye), taken at the Co $L_{3}$ edge, represent the magnetic response of the Co atoms only. The circles, taken at the Fe $L_{3}$ edge, represent the magnetic response of only the Fe cap layer. For both films, the coercive field is $10 \mathrm{G}$, and the loop has low squareness, suggesting multiple domains. The solid curve is the element specific hysteresis for the clean Co film $[7.0 \mathrm{~nm} \mathrm{Co} /$ $\mathrm{Si}(100)]$ from Fig. 2. 


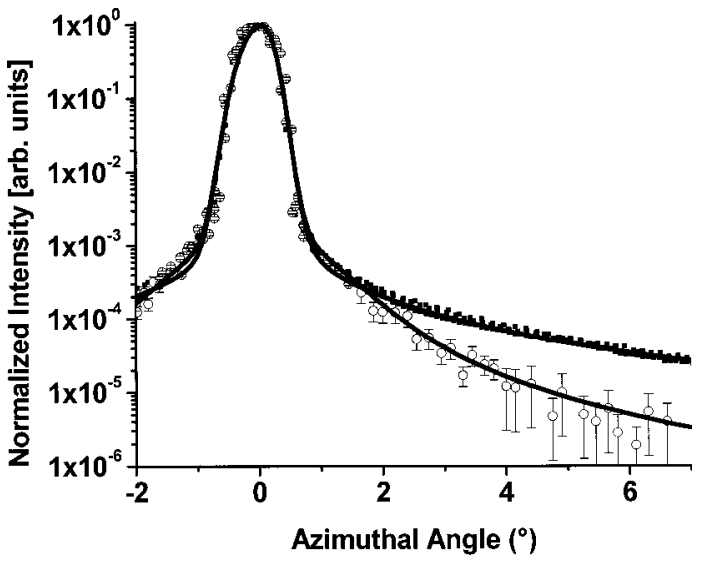

FIG. 4. DXRMS scans of chemical and magnetic-boundary morphology of the bare, clean Co film. Closed squares: charge scattering, open circles: charge-magnetic scattering. The curves are normalized at their peaks. The diffuse intensities reflect, respectively, the chemical-boundary and magneticboundary morphologies. The charge scattering shows a higher relative diffuse intensity, corresponding to a rougher chemical boundary. The lines are fits to the data. The chemical boundary over this $15 \mathrm{~mm} \times 1 \mathrm{~mm}$ area has rms roughness $\sigma=0.45 \pm 0.02 \mathrm{~nm}$ with a correlation length $\xi=17 \pm 2 \mathrm{~nm}$, and a Hurst parameter (describing the texture of the roughness) $h=0.4$ \pm 0.1 . The magnetic boundary has rms roughness $\sigma=0.43 \pm 0.03 \mathrm{~nm}$, with a correlation length $\xi=22 \pm 2 \mathrm{~nm}$, and a Hurst parameter $h=0.8 \pm 0.15$. The higher chemical-boundary roughness is mostly caused by a greater jaggedness.

the chemical and magnetic boundaries and that these results are very similar to those for Al-capped Co (Fig. 5). Hence, quenching by surrounding nonmagnetic atoms cannot be the cause of the difference between chemical and magnetic interfaces.

We fit the shape of the charge scattering with the DWBA to obtain exact measures of the roughness parameters of the chemical boundary. We fit the shape of the difference signal with the DWBA ${ }^{14}$ (without correcting for chemical/magnetic boundary morphology correlations as described earlier) to obtain qualitative measures of the roughness parameters for the magnetic boundary. The bare Co sample has a rms

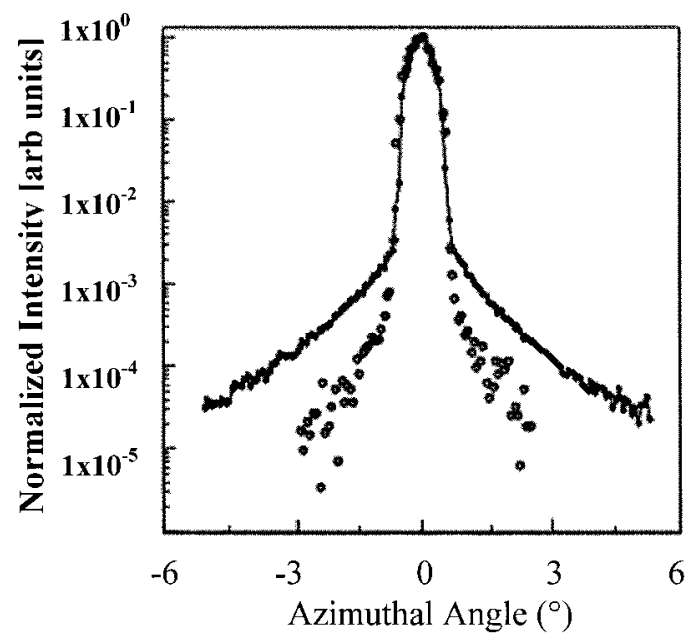

FIG. 5. DXRMS scans of the Al capped Co film. Closed circles: charge scattering; open circles: charge-magnetic scattering. The curves are normalized at their peaks. The charge scattering shows a higher relative diffuse intensity, corresponding to a rougher chemical boundary.

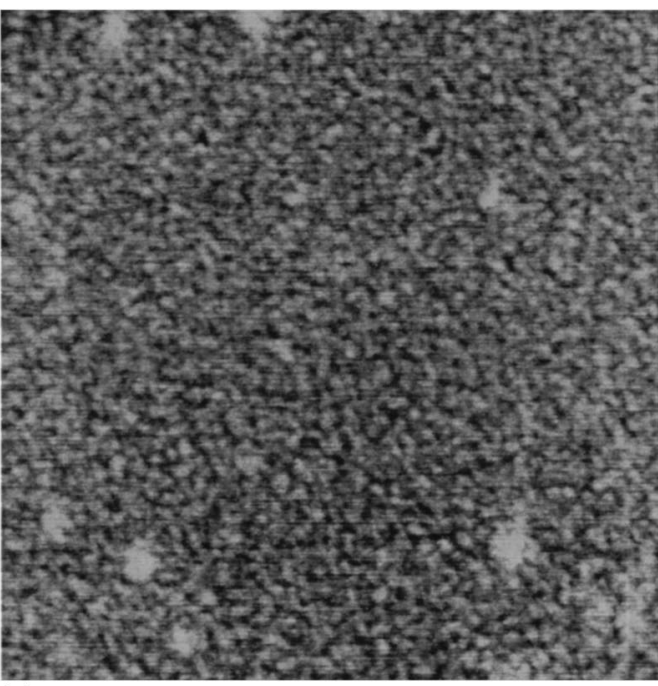

(a)

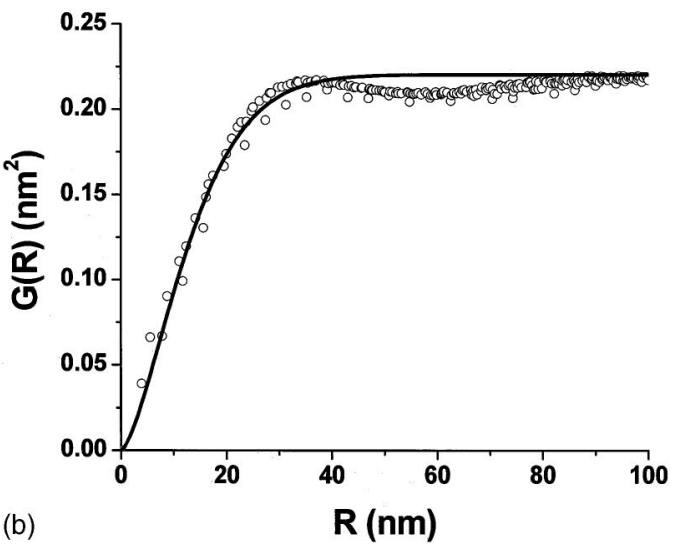

FIG. 6. (a) AFM image $(2 \mu \mathrm{m} \times 2 \mu \mathrm{m})$ of the bare Co surface $[7.0 \mathrm{~nm}$ $\mathrm{Co} / \mathrm{Si}(100)]$. The image was obtained in intermittent-contact mode using a carbon nanotube tip. The $z$ range is $5.0 \mathrm{~nm}$. It is expected that the surface is oxidized. (b) Comparison of height-height correlation functions. Open circles: $G(R)=\left\langle[z(R)-z(0)]^{2}\right\rangle$ calculated from raw AFM data from Fig. 6(a). Solid line: Fit to raw data using Eq. (5). The shape of $G(R)$ demonstrates a clear long-wavelength cutoff to the morphology. The slight dip in the measured $G(R)$ may be due a slightly granular structure or tip effects. Roughness parameterization indicates that over this $2 \mu \mathrm{m} \times 2 \mu \mathrm{m}$ area, the rms roughness, $\sigma$, is $0.34 \pm 0.02 \mathrm{~nm}$, the lateral correlation length, $\xi$, is 15 $\pm 1 \mathrm{~nm}$, and the Hurst parameter, $h$, is $0.75 \pm 0.1$. Oxidation might lead to the discrepancy in the rms roughness with the in situ x-ray fit in Fig. 4, though the lateral correlation length agrees well. The relative width the AFM tip compared to the x-ray wavelength obscures comparison of the Hurst parameter.

chemical surface roughness $\sigma=0.45 \pm 0.02 \mathrm{~nm}$ with a correlation length $\xi=17 \pm 2 \mathrm{~nm}$, and a Hurst parameter (describing the texture of the roughness) $h=0.4 \pm 0.1$. AFM analysis (Fig. 6) gives a rms roughness of $0.34 \pm 0.02 \mathrm{~nm}$, with a correlation length of $15 \pm 1 \mathrm{~nm}$. Figure $6(\mathrm{~b})$ shows the correlation function. This correlation function is calculated from the heights $z(R)$ of the AFM data by

$$
G(R)=\left\langle[z(R)-z(0)]^{2}\right\rangle \text {. }
$$

If one assumes that the surface is self-affine fractal one can use $C(R)$ as shown in Eq. (1). Then $C(R)$ and $G(R)$ are related by ${ }^{26}$

$$
G(R)=2 \sigma^{2}-2 C(R) .
$$




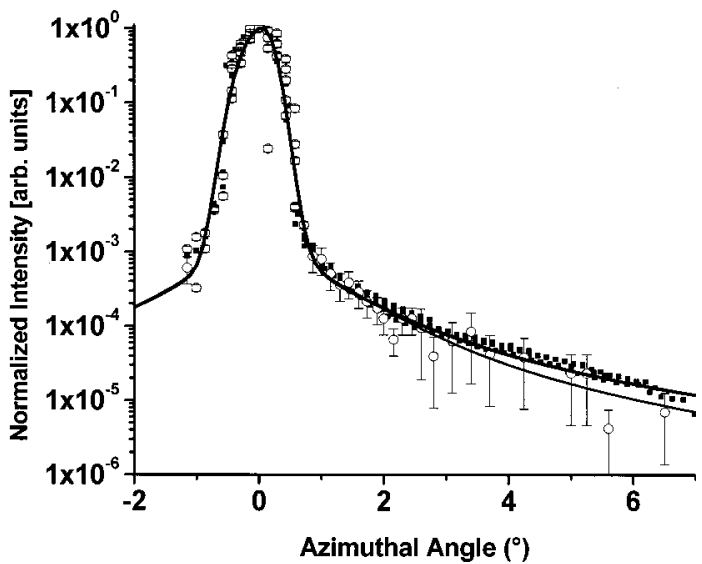

FIG. 7. DXRMS scans of the Fe-capped Co film, using the Co $L_{3}$ edge. Closed squares: charge scattering; open circles: charge-magnetic scattering. The charge and charge-magnetic profiles are identical within experimental error, indicating that the magnetic and chemical boundaries have the same roughness. The magnetic-boundary roughness has increased relative to that of the bare Co surface, while the chemical-boundary roughness has decreased. The lines are fits to the data. The rms boundary roughness of the Co boundary is $\sigma=0.36 \pm 0.03 \mathrm{~nm}$, with a correlation length $\xi=15 \pm 2 \mathrm{~nm}$, and a Hurst parameter $h=0.6 \pm 0.1$. The rms chemical-magnetic-boundary roughness of the Co film is $\sigma=0.35 \pm 0.02 \mathrm{~nm}$, with a correlation length $\xi$ $=17 \pm 8 \mathrm{~nm}$, and a Hurst parameter $h=0.6 \pm 0.2$.

Fits to $\Delta I$ give a rms magnetic surface roughness $\sigma$ $=0.43 \pm 0.03 \mathrm{~nm}$, with a correlation length $\xi=22 \pm 2 \mathrm{~nm}$, and a Hurst parameter $h=0.8 \pm 0.15$. The differences are primarily in the Hurst parameter. A Hurst parameter of 1 indicates smooth hills with little very-short-wavelength roughness, while Hurst parameters approaching zero correspond to surfaces that are very jagged on short length scales. The much larger Hurst parameter of the magnetic roughness suggests (see discussion later) that some short-wavelength roughness present at the chemical boundary is not present at the magnetic boundary.

The $\mathrm{Al}$ capped Co films had a rms chemical roughness of the Co layer of $\sigma=0.29 \mathrm{~nm}$, with a correlation length $\xi$ $=12.5 \pm 1.5 \mathrm{~nm}$, and a rms magnetic roughness of $\sigma$ $=0.15 \mathrm{~nm}$, with a correlation length $\xi=20 \pm 5 \mathrm{~nm} .{ }^{14}$ In these earlier experiments, best fits were obtained assuming the Hurst parameters to be the same, 0.5, for both magnetic and chemical boundaries. ${ }^{14}$

By using a magnetic-film capping layer, we can explore if the presence of nearby magnetic moments influences the roughness of the magnetic boundary. We can tune to a specific element by exploiting the element specificity of DXRMS. We performed DXRMS scans on the $\mathrm{Fe} / \mathrm{Co} / \mathrm{Si}$ sample at the $\mathrm{Co}_{2}$ edge $(775 \mathrm{eV})$. This measurement primarily examines the Co boundary of the $\mathrm{Fe} / \mathrm{Co}$ interface because the thickness of the Co film leaves the lower interface $(\mathrm{Co} / \mathrm{Si})$ effectively invisible to the beam. The results are shown in Fig. 7. For this film structure $(\mathrm{Fe} / \mathrm{Co})$ the difference between magnetic and charge scattering of the Co boundary at the $\mathrm{Fe} / \mathrm{Co}$ interface has largely disappeared, in contrast to the measurements on the bare or Al-capped Co films. We expect on the basis of these results that the $\mathrm{Fe}$ moments at the $\mathrm{Co} / \mathrm{Fe}$ interface are aligned with the bulk moments. Figure 7 confirms this expectation. We expect the free-surface

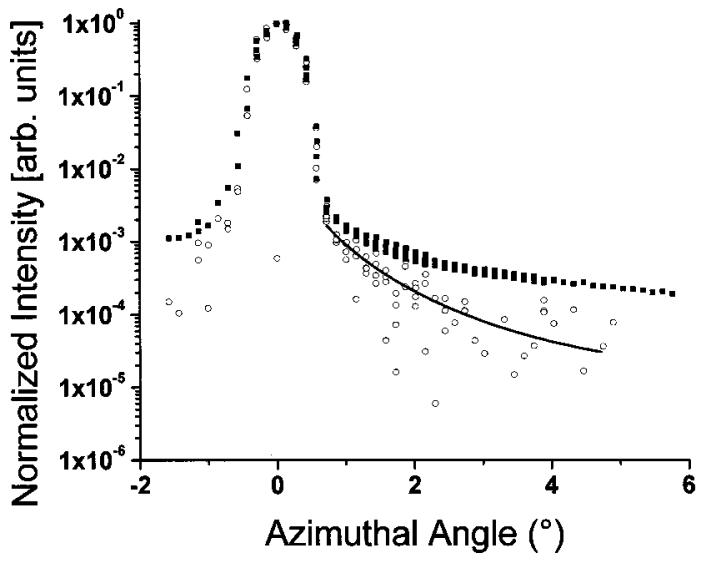

FIG. 8. DXRMS scans of the Fe-capped Co film, using the Fe $L_{3}$ edge. Closed squares: charge scattering; open circles: charge-magnetic scattering. The charge and charge-magnetic profiles are different within the experimental error. The line through the charge-magnetic scattering is a guide to the eye.

Fe moments to behave the same way as those of a bare Co film. We test this expectation by using the $\mathrm{Fe} L_{3}$ edge (710 $\mathrm{eV})$. The Fe film is thin enough so that we should see both the $\mathrm{Fe} / \mathrm{Co}$ and $\mathrm{Fe} /$ vacuum boundaries, with the latter seen much more strongly, however. A difference between the magnetic and charge scattering once again appears (Fig. 8), with the Fe magnetic boundary smoother than its chemical boundary. Figure 8 shows that the same overall behavior as that seen in bare Co occurs at the surface of the Fe film. The Fe-capped Co film has a rms chemical-boundary roughness of the Co boundary of $\sigma=0.36 \pm 0.03 \mathrm{~nm}$, with a correlation length $\xi=15 \pm 2 \mathrm{~nm}$, and a Hurst parameter $h=0.6 \pm 0.1$. AFM analysis (Fig. 9) gives a rms surface roughness of the Fe layer of $0.26 \pm 0.02 \mathrm{~nm}$, and a correlation length of 15 $\pm 3 \mathrm{~nm}$. Fits to $\Delta I$ give a rms magnetic-boundary roughness of the Co film of $\sigma=0.35 \pm 0.02 \mathrm{~nm}$, with a correlation length $\xi=17 \pm 8 \mathrm{~nm}$, and a Hurst parameter $h=0.6 \pm 0.2$,

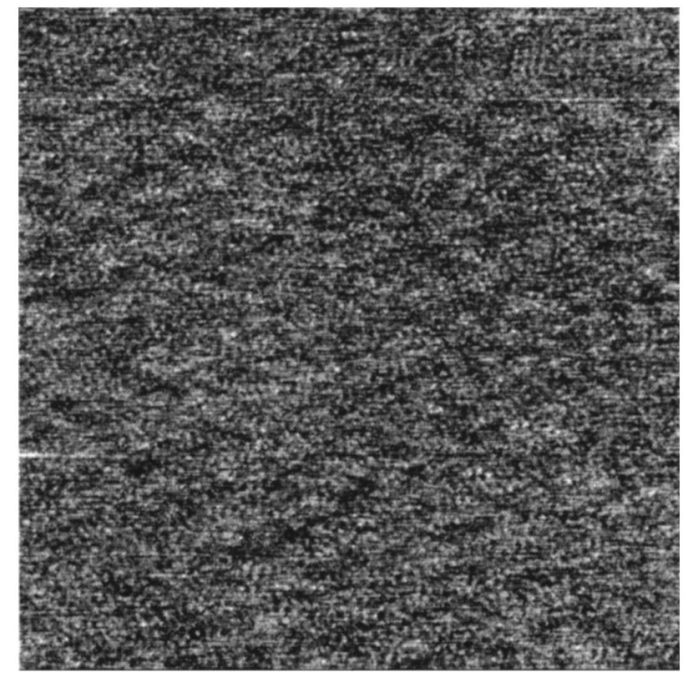

FIG. 9. AFM scan $(2 \mu \mathrm{m} \times 2 \mu \mathrm{m})$ of the Fe layer in the Fe/Co sample [2.0 $\mathrm{nm} \mathrm{Fe} / 7.0 \mathrm{~nm} \mathrm{Co} / \mathrm{Si}(100)]$. The image was obtained in intermittent-contact mode using a carbon nanotube tip. The $z$ range is $5.0 \mathrm{~nm}$. The rms roughness, averaged over a $2 \mu \mathrm{m} \times 2 \mu \mathrm{m}$ area, is $0.26 \mathrm{~nm}$. 
very similar to those for the chemical-boundary roughness of the Co film. It is clear that the chemical and magnetic boundaries have come into concordance: the chemical boundary has become somewhat less rough and somewhat less jagged, while the magnetic boundary has become rougher, especially in terms of jaggedness.

\section{DISCUSSION}

We have performed element-specific hysteresis measurements using specular XRMS, magnetic- and chemicalboundary roughness measurements using DXRMS, and surface roughness measurements using AFM on three types of samples to order to assess possible mechanisms that contribute to differences in the roughnesses of the magnetic and chemical boundaries.

Element-specific hysteresis on the bare Co sample shows a low coercivity of $12 \mathrm{G}$ and shows high squareness. We define squareness as $M_{B=0} / M_{\text {saturation. Thus these Co films }}$ are likely single-domain. The Fe-capped samples also have low coercivity $\left(H_{c}=10 \mathrm{G}\right)$, but lower squareness, suggesting multiple domains. The low coercivity values indicate that the films have a low defect density, as lower coercivity generally indicates fewer pinning centers for magnetic domains. ${ }^{28}$

All previous DXRMS measurements have used samples capped with a nonmagnetic material and have shown differences between chemical and magnetic scattering. ${ }^{14,17,18}$ All these measurements were interpreted as having a smoother magnetic boundary, i.e., one with a reduced shortwavelength roughness. The behavior of a bare Co film described here is similar to the earlier results using a nonmagnetic capping layer. The behavior of Fe-capped Co films is markedly different.

Accepting straightforwardly the conclusions obtained from fitting the charge (average) scattering and the chargemagnetic (difference) scattering as described earlier, one concludes that the magnetic and chemical boundaries for the bare Co film are quite different on the local scale, even though the rms roughness and in-plane correlation length of the magnetic boundary are roughly the same as those of the chemical boundary. The difference appears in the Hurst parameter, which is much greater for the magnetic boundary than the chemical boundary, implying that the former has considerably less short-wavelength roughness than the latter. However, as the dominant contribution to the differencescattering diffuse intensity is the crosscorrelation between the morphologies of the chemical and magnetic boundaries, directly interpreting the fit parameters is somewhat problematic. We discuss this situation in more detail later.

An Fe capping layer removes the qualitative morphological differences observed in the magnetic and chemical boundaries for clean Co films or Co films capped with nonmagnetic films. Physically, for bare surfaces or magnetic films capped with nonmagnetic materials, moments at the roughest parts of the interface do not track the applied magnetic field as the bulk moments do. While it is not certain that all Co moments follow the applied magnetic field for
Fe-capped Co films, moments track better than they do on bare or nonmagnetically capped films.

As mentioned in the introduction, in previous work on Al-capped samples, three models for this lack of tracking by "exposed" moments were proposed. With the present work we have clearly eliminated pinning by nonmagnetic atoms at the interface as the cause for the different morphologies of magnetic and chemical boundaries. The two remaining models may still be consistent with the results.

The "fixed spin" model focuses on anisotropy effects. The disruption of the crystalline periodicity at the interface could create a magnetic anisotropy that might cause the moments to align preferentially out of the plane, with an energy barrier for aligning the moments parallel to the interface. Both theory and experiment have shown ${ }^{29-31}$ that the change in electron orbitals at a surface or interface can cause a magnetic anisotropy that may produce out-of-plane moment orientation. Capping layers that are chemically dissimilar would leave the moments fixed out of plane, with the fields we use insufficient to align them. When the cap layer is chemically similar to Co (e.g., an Fe cap), one could argue that this similarity makes the magnetic anisotropy weaker and the energetic barriers lower, and thus the moments can align. Estimates of the anisotropy field for $2 \AA$ Co films in multilayers vary from 10 to $50 \mathrm{kOe}^{32}$

The fixed spins model would apply for any magnetic/ nonmagnetic or magnetic/vacuum interface, irrespective of its roughness. In other words, one would expect a "dead layer," for which the spins do not respond to the applied field, even for a smooth surface-perhaps especially for a smooth surface. The effect, for a smooth surface, would be simply to reduce the overall magnetic signal from the film, something very hard to measure. It is not clear how sharp asperities should affect the anisotropy. More symmetries are broken for a moment well out of the average plane, but it is hard to see how the energy barrier for alignment could increase under those circumstances. A reductio-ad-absurdum argument would suggest that the spin of a solitary atom on top of a narrow peak would be fixed most rigidly of all spins. Hence for the fixed-spins model, reducing interface roughness may not have the result of better coupling to the bulk moments.

The flopping spins model, on the other hand, focuses on the reduced coordination of surface or interface moments, which could cause the moments to become paramagnetic and controlled in their orientation by the thermal energy, $h T$. The moments will align somewhat with the applied field. At room temperature, however, no realistic field can align any appreciable fraction of the moments. ${ }^{33}$ The flopping spin model quite straightforwardly incorporates the observed roughness wavelength dependence. For a moment with fewer neighbors, the ferromagnetic exchange interactions get weaker, and it becomes more like a paramagnet. Thus, the paramagnetic effects will be strongest for the most isolated spins.

Recent work by Willis has shown that ultrathin films of magnetic materials have drastically lower Curie temperatures than the corresponding bulk material. ${ }^{34}$ For Co, the Curie temperature decreases from a bulk value of $1388 \mathrm{~K}$ to $300 \mathrm{~K}$ for a 2-ML-thick film. ${ }^{35}$ The highest asperities of a 
rough interface may behave like an extremely thin film, even though they are in contact with a magnetic bulk. If the Curie temperature of these rough regions is below $300 \mathrm{~K}$, the entire magnetic boundary could behave like a paramagnet.

The similarity of the magnetic and chemical roughnesses of the Co interface for the Fe capped sample $(2.0 \mathrm{~nm} \mathrm{Fe} / 7.0$ $\mathrm{nm} \mathrm{Co} /\langle 100\rangle \mathrm{Si})$ can readily be explained by the floppingspins model. Because Fe is strongly magnetic, the Fe at the interface compensates for the loss of magnetism that a bare Co surface with flopping spins experiences and thus allows the moments sitting at asperities to align better than they would have for a bare surface or one capped by a nonmagnetic film.

Flopping spins would, in our opinion, be present even if a crystalline anisotropy causes the spins at the surface/ interface to desire to be fixed out of plane. In other words, roughness ought to make the spins at asperities flop more readily. Hence, independent of whether an anisotropy fixes spins at an interface, the magnetic-boundary roughness ought still be controlled by the degree to which spins have become paramagnetic. If one were to assume that all nonfully coordinated atoms in the interface have moments that do not participate in the bulk magnetic properties of the film, then one estimates, for the interface roughnesses we measure, that over $10 \%$ of the moments do not participate in the bulk magnetic behavior of a $7 \mathrm{~nm}$ Co film. This estimate is an upper bound on the number of moments that do not contribute. As the film becomes thinner, a larger percentage of moments is lost to the bulk magnetism.

Returning now to the interpretation of the diffuse intensity in the difference scattering in terms of greater smoothness of the magnetic boundary, we do have some clear results from our experiments. For the Fe-capped Co film, the shapes of the charge and charge-magnetic diffuse intensities are the same, meaning that the charge and magnetic interfaces are identical, and thus perfectly correlated. For the bare Co film, the shape of the charge and charge-magnetic diffuse intensities are not the same. The degree of correlation between the charge and magnetic interfaces therefore becomes unclear. Crosscorrelations between interfaces discriminate against short-wavelength roughness if the longer-wavelength correlation between the interfaces is less than perfect. A larger $h$, implying a loss of short-wavelength roughness, could thus be due simply to the low-pass-filter nature of crosscorrelation if there is reduced longer-wavelength correlation between the interfaces. It might be expected, however, and has been found in other thin-film systems, ${ }^{36}$ that vertical correlation lengths are similar in magnitude to in-plane correlation lengths. The in-plane correlation lengths in this system are on the order of $20 \mathrm{~nm}$. The separation of the chemical and magnetic boundaries is likely on the order of $1 \mathrm{~nm}$, and obviously less than the thickness of the film. Thus, there should not be significant loss of longer-wavelength correlation between the chemical and magnetic boundaries. The larger value of the Hurst parameter obtained for the magnetic scattering thus does suggest that the magnetic boundary is less jagged than the chemical boundary, but a quantification of the degree of smoothening requires that details of the crosscorrelation be understood. An obvious next step is to predict explicitly the correlation from a model relating the chemical and magnetic boundaries. We expect such a model will predict a less jagged magnetic boundary. All physical models for the magnetic boundary predict it to be smoother than the chemical boundary.

In summary, we believe that we observe a smoother magnetic boundary and that the flopping-spins model is the ultimate cause for the loss of moments in the total magnetization of a film if the interface is rough, although we cannot prove the latter at this stage. Additional experiments might shed further light on this issue. If the flopping-spins model applies, the magnetic roughness ought to increase at sufficiently low temperatures. Our limited attempts to make measurements as low as $100 \mathrm{~K}$ for $\mathrm{Al} / \mathrm{Co}$ films have so far produced no differences from those at $300 \mathrm{~K}$. We will extend these experiments, but it may simply not be possible to align flopping spins at a rough interface because of the lowered Curie temperatures discussed earlier.

The asymmetry ratio contains additional information that we have not, as yet, exploited. As discussed earlier, in the limit that a film is nonmagnetic or for some reason entirely pinned, the asymmetry ratio is zero, i.e., there is no change in the intensity when the direction of the field is reversed. Conversely, in the limit in which all the spins follow the applied field, the asymmetry ratio is a maximum, i.e., the difference is as large as it can be. If we consider two films of equal thickness, we can ask how varying the roughness will affect the asymmetry ratio within the confines of our simple models.

In the flopping-spins model for a perfectly smooth film, the maximum number of spins can follow the applied field. Any addition of roughness will decrease this number (as the number of neighbors for a moment at an asperity decreases) and, hence, the asymmetry ratio. For the surface pinning model, we can argue that the asymmetry ratio should be approximately independent of increasing roughness. It is apparent in the surface pinning model that a perfectly smooth film will have spins that do not contribute to the bulk magnetism. There will be some depth to which the spins are pinned, yielding a "dead" layer. If roughness is added we expect the dead-layer thickness to change only slightly, if at all. If it changes, we expect a decrease as spins pinned at differing angles will tend to cancel each other. Thus, we expect either no change or a slight increase in the asymmetry ratio with increasing roughness. We have not yet performed these experiments, as critical attention must be paid to angles of incidence to avoid misleading effects.

\section{CONCLUSION}

We have made measurements to quantify the morphology of magnetic and chemical boundaries in internal interfaces between magnetic and nonmagnetic metals. Our results support the concept of a magnetic boundary lying in the magnetic material "below" the chemical boundary, and one that is smoother than the chemical boundary. Some volume of a magnetic film bordered by a nonmagnetic film does not respond to applied magnetic fields that are at least ten times the strength needed to align the bulk moments. Even at rms 
roughnesses as low as $0.2-0.3 \mathrm{~nm}$, there is a non-negligible number of magnetic moments that do not contribute to the overall magnetization, perhaps as much as $10 \%$ for the thicknesses of the magnetic films we use. It is so far not possible to distinguish uniquely whether the "lost" moments are pinned in a fixed direction or are flopping because of their thermal energy. Measurements on a clean surface allow us to eliminate a third choice, chemical quenching of moments by the capping layer. We believe that careful measurements of the asymmetry ratio for samples with different surface roughnesses will identify the correct model. The answer could allow improved engineering of these films to enhance their magnetic properties, and, in fact, help determine if extreme smoothness is a meaningful goal in optimizing interface magnetic properties in magnetoelectronic devices.

\section{ACKNOWLEDGMENTS}

The authors thank F. Liu and F. Himpsel for useful discussions and K. Moloni at Piezomax Technologies for use of its AFM and carbon nanotube probes. This work was supported by Seagate, ONR, and AFOSR, and conducted in part at the Synchrotron Radiation Center, University of Wisconsin-Madison. The NSF supports the SRC under Award No. DMR-0088449.

${ }^{1}$ F. Nguyen-Van-Dau, M. Sussiau, A. Schuhl, and P. Galtier, J. Appl. Phys. 81, 4482 (1997).

${ }^{2}$ A. Encinas, F. Nguyen-Van-Dau, M. Sussiau, A. Schuhl, and P. Galtier, Appl. Phys. Lett. 71, 3299 (1999).

${ }^{3}$ W. H. Meiklejohn and C. P. Bean, Phys. Rev. 105, 904 (1957).

${ }^{4}$ K. Takano, R. H. Kodama, A. E. Berkowitz, W. Cao, and G. Thomas, Phys. Rev. Lett. 79, 1130 (1997).

${ }^{5}$ P. M. Levy, S. Zhang, and A. Fert, Phys. Rev. Lett. 65, 1643 (1990).

${ }^{6}$ V. S. Speriosu, J. P. Nozieres, B. A. Gurney, B. Dieny, T. C. Huang, and H. Lefakis, Phys. Rev. B 47, 11579 (1993).

${ }^{7}$ E. E. Fullerton, D. M. Kelly, J. Guimpel, I. K. Schuller, and Y. Bruynseraede, Phys. Rev. Lett. 68, 859 (1992).

${ }^{8}$ M. Suzuki and Y. Taga, Phys. Rev. B 52, 361 (1995).

${ }^{9}$ L. Sheng, H. Y. Teng, and D. Y. Xing, Eur. Phys. J. B 10, 209 (1999).

${ }^{10}$ D. E. Joyce, C. A. Faunce, P. J. Grundy, B. D. Fulthorpe, T. P. A. Hase, I. Pape, and B. K. Tanner, Phys. Rev. B 58, 5594 (1998).
${ }^{11}$ R. Schad, P. Belien, G. Verbanck, V. V. Moshchalkov, Y. Bruynserade, H. E. Fischer, S. Lefebvre, and M. Bessiere, Phys. Rev. B 59, 1242 (1999).

${ }^{12}$ J. W. Cable, M. R. Khan, G. P. Felcher, and I. K. Schuller, Phys. Rev. B 34, 1643 (1986)

${ }^{13}$ M. J. Pechan, J. F. Ankner, C. F. Majkrazk, D. M. Kelly, and I. K. Schuller, J. Appl. Phys. 75, 6178 (1994).

${ }^{14}$ J. F. MacKay, C. Teichert, D. E. Savage, and M. G. Lagally, Phys. Rev. Lett. 77, 3925 (1996).

${ }^{15}$ E. Y. Vedmednko, H. P. Oepen, A. Ghazali, J.-C. S. Levy, and J. Kirschner, Phys. Rev. Lett. 84, 5884 (2000).

${ }^{16}$ D. Zhao, F. Liu, D. L. Huber, and M. G. Lagally, Phys. Rev. B 62, 11316 (2000).

${ }^{17}$ J. W. Freeland, V. Chakarian, K. Bussmann, Y. U. Idzerda, H. Wende, and C.-C. Kao, J. Appl. Phys. 83, 6290 (1998).

${ }^{18}$ C. S. Nelson, G. Srajer, J. C. Lang, C. T. Venkataraman, S. K. Sinha, H. Hashizume, N. Ishimatsu, and N. Hoshito, Phys. Rev. B 60, 12234 (1999).

${ }^{19}$ R. M. Osgood III, S. K. Sinha, J. W. Freeland, Y. U. Idzerda, and S. D. Bader, J. Appl. Phys. 85, 4619 (1999).

${ }^{20}$ T. P. A. Hase, I. Pape, B. K. Tanner, H. Dürr, E. Dudzik, G. van der Laan, C. H. Marrows, and B. J. Hickey, Phys. Rev. B 61, 3792 (2000).

${ }^{21}$ T. Salditt, T. H. Metzger, and J. Peisel, Phys. Rev. Lett. 73, 2228 (1994).

${ }^{22}$ A. T. Macrander, C. Liu, R. Csencsits, R. Cook, M. Kirk, and R. Headrick, Physica B 283, 157 (2000).

${ }^{23}$ M. Rauscher, T. Salditt, and H. Spohn, Phys. Rev. B 52, 1685 (1995)

${ }^{24}$ C.-C. Kao et al., Phys. Rev. B 50, 9599 (1994).

${ }^{25}$ T. Larsen, K. Moloni, F. Flack, M. Eriksson, M. G. Lagally, and C.T. Black, Appl. Phys. Lett. 80, 1996 (2002).

${ }^{26}$ S. K. Sinha, E. B. Sirota, S. Garoff, and H. B. Stanley, Phys. Rev. B 38, 2297 (1988)

${ }^{27}$ J. P. Hannon, G. T. Trammell, M. Blume, and D. Gibbs, Phys. Rev. Lett. 61, 1245 (1988)

${ }^{28}$ K. N. Altmann et al., Mater. Res. Soc. Symp. Proc. 570, 141 (1999), and references therein.

${ }^{29}$ D. Weller et al., Phys. Rev. Lett. 75, 3752 (1995).

${ }^{30}$ J. Stöhr and H. König, Phys. Rev. Lett. 75, 3748 (1995).

${ }^{31}$ H. A. Durr, G. Y. Guo, G. van der Laan, J. Lee, G. Lauhoff, and J. A. C. Bland, Science 277, 213 (1997).

${ }^{32}$ D. Weller, Y. Wu, J. Stöhr, M. G. Samant, B. D. Hermseier, and C. Chappert, Phys. Rev. B 49, 12888 (1994).

${ }^{33}$ D. Jiles, Introduction to Magnetism and Magnetic Materials (Chapman \& Hall, London, 1991).

${ }^{34}$ R. Zhang and R. F. Willis, Phys. Rev. Lett. 86, 2665 (2001), and references therein.

${ }^{35}$ C. M. Schneider, P. Bressler, P. Schuster, J. Kirschner, J. J. de Miguel, and R. Miranda, Phys. Rev. Lett. 64, 1059 (1990).

${ }^{36}$ Y. H. Phang, D. E. Savage, R. Kariotis, and M. G. Lagally, J. Appl. Phys. 74, 3181 (1993) 